\title{
Radiation induced small bowel "web" formation is associated with acquired microvascular dysfunction
}

\author{
O A Hatoum, D G Binion, S A Phillips, C O'Loughlin, R A Komorowski, \\ D D Gutterman, M F Otterson
}

Background and aims: Radiation therapy of abdominal and pelvic solid tumours results in late intestinal toxicity of a severe nature in approximately $5 \%$ of cases. These manifestations may include ischaemia and stricture formation, which may present as "webs". These webs are likely to play a role in the pathogenesis of recurrent bowel obstruction. The mechanisms of microvascular injury to the bowel in the setting of radiation have not been defined. We hypothesised that microvascular dysfunction with impaired vasodilation to acetylcholine (Ach) would be an acquired pathophysiological abnormality in radiation and "web" formation.

Methods: A 40 year old patient treated with radiation, two years previously, for an anal squamous cell cancer presented with recurrent small bowel obstruction. "Webs" in the distal ileum were detected using wireless capsule endoscopy, after small bowel barium radiographs failed to demonstrate a lesion. Following resection, freshly isolated 50-150 $\mu \mathrm{m}$ diameter arterioles from the "web" and adjacent normal calibre bowel were analysed with histology and microvessel physiological studies.

Results: After constriction (30-50\%) with endothelin, dilation to graded doses of Ach $\left(10^{-9}-10^{-4} \mathrm{M}\right)$ was observed in vessels dissected from the stricture and the adjacent normal calibre area. Ach dilation was reduced in vessels from "web" (mean diameter 7 (2)\%; $\mathrm{n}=3, \mathrm{p}<0.01$ ) compared with the adjacent unaffected bowel (mean diameter $85(5) \%$ ). Dihydroethidine and dichlorofluorescein diacetate intravital staining demonstrated increased reactive oxygen species production in microvessels from "web" compared with adjacent normal calibre bowel. Histology from the strictured bowel demonstrated narrowing of the arterial lumen due to intimal and muscularis propria fibrosis, with endothelial preservation.

Conclusions: External radiation is associated with acquired microvascular endothelial dysfunction and "web" formation in the small bowel.

S mall intestine "webs" are increasingly appreciated to play a role in a spectrum of small bowel pathology, including occult gastrointestinal bleeding, enteropathy, and intermittent partial small bowel obstruction. The prevalence of small bowel webs is not known but autopsy series suggest that specific adult populations may demonstrate stricture related lesions in $1.5-9 \%$ of individuals. ${ }^{1-3}$

Late radiation injury of the small intestine may take years, with a median of approximately 8-12 months before the injury becomes apparent. ${ }^{45}$ The clinical signs and symptoms of chronic radiation intestinal injury may be multifactorial in aetiology. The chief problems that may require major medical or surgical intervention are intestinal obstruction, severe bleeding, fistula formation, and intractable diarrhoea. ${ }^{4-6}$ The radiological picture of late radiation injury is of fibrosis and ischaemia. These lesions are frequently missed in standard endoscopy and contrast studies of the upper and lower gastrointestinal tract. Capsule endoscopy is a new method that permits the endoscopic examination of the mucosa of the entire small bowel. The advent of wireless capsule endoscopy has increased diagnostic yield in patients with negative contrast studies. Using this technique, mucosal "webs" may be found on review of the video study or by capsule retention.

Here, we describe a case of radiation ileal "web", diagnosed by capsule endoscopy, and its association with local acquired microvascular dysfunction and impaired vasodilator capacity.

\section{CASE REPORT}

A 40 year old female, with a medical history significant for external beam radiation treatment of a T2NOM0 squamous cell anal cancer, was evaluated for symptoms of small bowel obstruction. She had received anal canal/inguinal node irradiation of 3060 centigray (cGy) in 17 daily fractions (18 megavoltage $(\mathrm{MV}) \times$ rays) with reduced field anal irradiation of $1980 \mathrm{CGy}$ ( $18 \mathrm{MV} x$ rays) and $1980 \mathrm{cGy}$ bilateral inguinal node irradiation (9 MeV electrons) in 11 fractions, completed 18 months previously. This brought the tumour dose to $5040 \mathrm{cGy}$. The patient received concurrent chemotherapy. Within 12 months of the radiation treatment, she experienced small bowel obstruction due to stricture formation and rectal sphincter damage requiring terminal ileal resection, primary anastomosis, and diverting sigmoid colostomy. During this procedure the remaining small intestine was carefully assessed for the presence of occult strictures using intraoperative passage and withdrawal of a balloon catheter inflated to a diameter of $2-2.5 \mathrm{~cm}$ through an enterotomy. ${ }^{7}$ Six months later, she again experienced symptoms of partial small bowel obstruction, which led to the current admission. Radiographic studies (plain radiographs, computed tomography, small bowel barium radiographs) and endoscopic studies (upper endoscopy, stoma colonoscopy) were non-diagnostic. Wireless capsule enteroscopy (fig 1A) resulted in capsule retention (fig 1B) at an ileal web stricture (fig lC), which was thought to be the cause of her symptoms. The patient underwent exploratory laparotomy during which two webs in a $25 \mathrm{~cm}$ ileal segment were identified and resected. The resected tissues, including the "webs" and the adjacent normal calibre intestine, were used for physiological studies and pathological analysis.

Abbreviations: ROS, reactive oxygen species; Ach, acetylcholine; DCF$\mathrm{DA}$, dichlorofluorescein diacetate; HE, hydroethidene; cGy, centigray; $\mathrm{O}_{2}{ }^{--}$, superoxide; $\mathrm{MV}$, megavoltage 

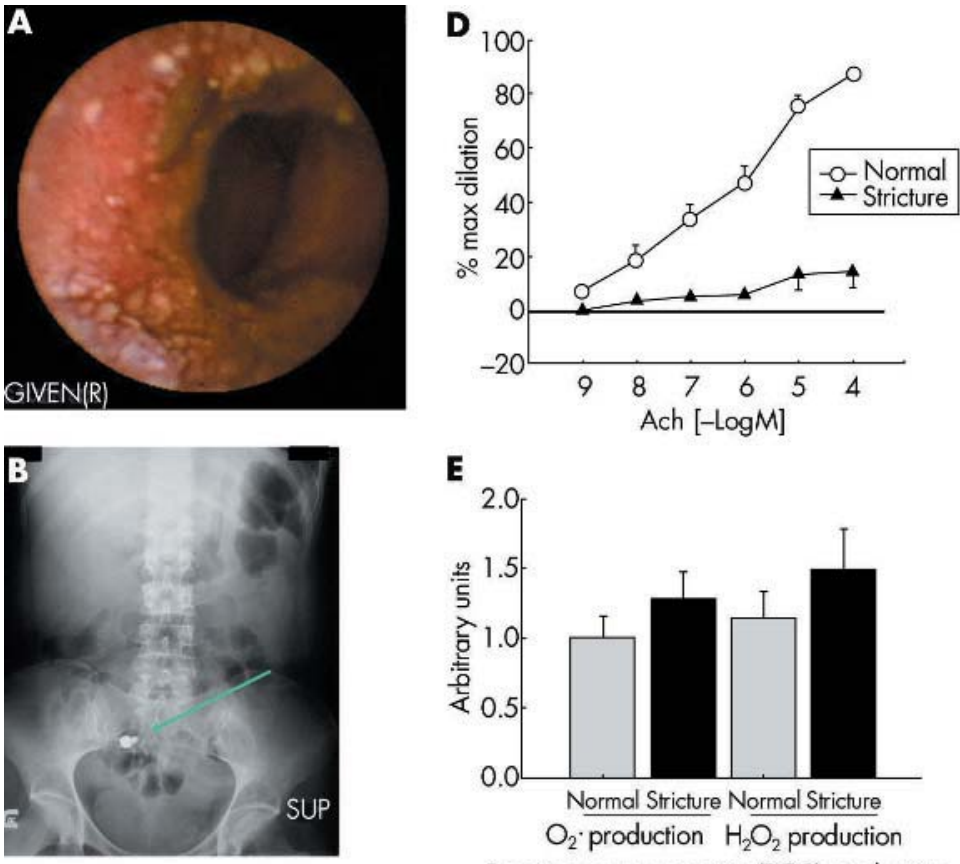

E

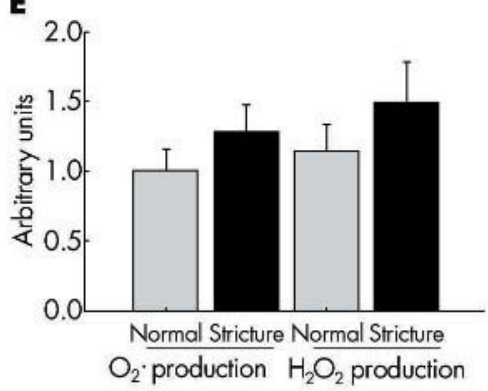

Reactive oxygen species (ROS) production from isolated microvessels
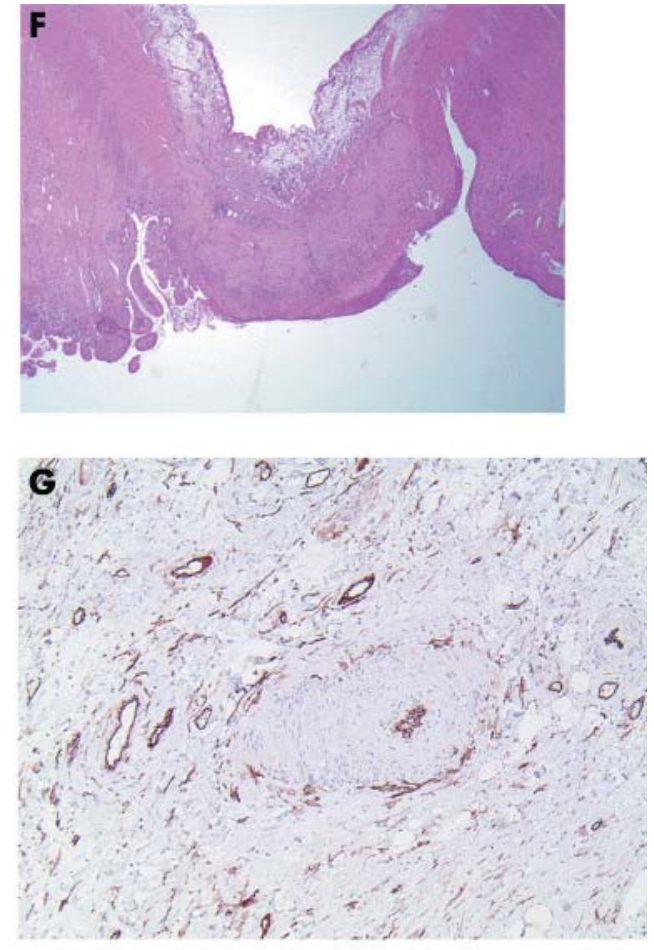

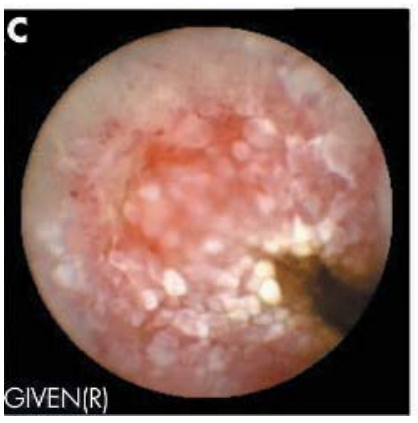

Figure 1 Microvascular dysfunction is associated with radiation enteritis induced intestinal strictures. (A) Wireless capsule endoscopic appearance of radiated non-stenotic ileum. (B) Abdominal radiograph demonstrating capsule retention. (C) Capsule endoscopic appearance of an ileal "web" following radiation therapy. (D) Acetylcholine (Ach) induced dilation of microvessels from radiation induced intestinal stricture and adjacent normal calibre intestine. (E) Reactive oxygen species (ROS) production from intestinal arterioles isolated from "web" and normal calibre bowel. (F) Histology of radiation induced stricture. (G) Endothelial staining with CD34 immunolocalisation in the radiation induced intestinal web, demonstrating preservation of the endothelial layer in stricture microvessels. Consent from the patient regarding the publication of this case report and the images was obtained.

\section{Material and methods}

The Medical College of Wisconsin's Institutional Review Board approved all protocols. Mucosal gut arterioles were dissected from the full thickness specimen from both strictured and normal calibre small bowel. Following resection, tissue samples were preserved as reported previously. ${ }^{8}$

\section{Microvessels physiology studies}

Video microscopy was performed as previously reported. ${ }^{8}$ Briefly, isolated microvessels from the strictured and normal calibre small intestine were carefully dissected from the submucosal surface of the bowel tissue and transferred to a $20 \mathrm{ml}$ organ chamber containing Krebs solution of the following composition (in $\mathrm{mmol} / \mathrm{l}$ ): $\mathrm{NaCl} 118, \mathrm{KCl} 4.7$, $\mathrm{CaCl}_{2} 2.5, \mathrm{KH}_{2} \mathrm{PO}_{4}$ 1.2, $\mathrm{MgSO}_{4} 1.2, \mathrm{NaHCO}_{3} 20, \mathrm{Na}_{2} \mathrm{EDTA}$ 0.026 , and glucose 11; pH 7.4. Each end of the arteriole was secured to a separate glass micropipette $(25-50 \mu \mathrm{m}$ internal diameter) filled with Krebs buffer ${ }^{9}$ and transferred to the stage of an inverted microscope (CK2; Olympus, Melville, New York, USA) coupled to a CCD camera (WV-BL200; Panasonic, Secaucus, New Jersey, USA) and video micrometer (VIA-100K; Boeckeler Instruments Inc., Tucson, Arizona, USA). Internal vascular diameters were measured throughout the experiment with a manually adjusted video microscope, as described previously. ${ }^{9}$ Micropipettes were connected to a hydrostatic reservoir at a final pressure of $60 \mathrm{~mm} \mathrm{Hg}$. The chamber solution was continuously recirculated at $30 \mathrm{ml} / \mathrm{min}$, aerated with $20 \% \mathrm{O}_{2}, 5 \% \mathrm{CO}_{2}$, and $75 \%$ $\mathrm{N}_{2}$, and warmed to $37^{\circ} \mathrm{C}$ by an external heat changer. All pharmacological agents were added to the external bathing solution.

After a 60 minute stabilisation period, vessels were constricted to $30-50 \%$ of maximal diameter (at $60 \mathrm{~mm} \mathrm{Hg}$ ) with administration of endothelin- $1\left(10^{-10}\right.$ to $\left.5 \times 10^{-10} \mathrm{M}\right)$. Vascular diameter responses to cumulative logarithmic increases in the concentration of acetylcholine (Ach) $\left(10^{-9}\right.$ to $10^{-4} \mathrm{M}$ ) in the external bathing media were examined. At the end of each experiment, passive vessel diameter was determined by adding papaverine $\left(10^{-4} \mathrm{M}\right)$. Addition of pharmacological agents produced less than $1 \%$ change in the volume of the circulating bath. All chemicals were obtained from Sigma Chemical Co. (St Louis, Missouri, USA).

\section{Fluorescence detection of reactive oxygen species (ROS)}

The cell permeable dye dihydroethidine (HE; Molecular Probes, Eugene, Oregon, USA) was used to evaluate the production of superoxide $\left(\mathrm{O}_{2}{ }^{--}\right)$. In the presence of 
superoxide, HE is oxidised to ethidium bromide, which remains trapped within the cell. ${ }^{10}$ Ethidium bromide fluorescence was excited by light at a wavelength of $488 \mathrm{~nm}$ with an emission spectrum of $620 \mathrm{~nm}$, and examined under confocal microscopy equipped with a krypton/argon laser fluorescence microscope (Nikon Eclipse, TE 200). A freshly isolated artery from normal calibre gut was used as a control to adjust for laser settings. Those settings were maintained constant throughout the remained of the experiment. The fluorescence intensity/vascular area ratio of the central portion of the vessel was normalised to that obtained from the control vessel using the public domain NIH Image program (developed at the US National Institutes of Health and available on the internet at http://rsb.info.nih. gov/nih-image/). The ratio of the fluorescence intensity between experimental vessel and control vessel was compared.

To evaluate the in situ production of all ROS (superoxide, hydrogen peroxide, and peroxynitrite), vessels were loaded with $5 \times 10^{-6} \mathrm{M}$ dichlorofluorescein diacetate (DCF-DA). DCF-DA oxidises rapidly to the highly fluorescent 2', $7^{\prime}$-dichlorofluorescein in the presence of $\operatorname{ROS}^{810}$ and was visualised on a fluorescence microscope. Captured digital images were analysed as described above.

\section{Tissue histology and immunostaining}

Paraffin embedded sections of the strictured and normal calibre intestine were examined under light microscopy with haematoxylin-eosin staining. The presence of an intact endothelial monolayer was assessed using CD 34 antibody immunodetection (DakoCytomation, Carpinteria, California, USA) with horseradish peroxide resolution.

\section{Materials}

Endothelin-1 (Peninsula Laboratories, Inc, San Carlos, California, USA) was prepared in saline with $1 \%$ bovine serum albumin. Other agents were prepared in distilled water. Final molar concentrations of agents in the organ chambers are reported. None of the pharmacological antagonists produced significant changes in baseline microvessels diameter.

\section{Results}

Microvessels from the stricture and macroscopic intervening normal areas of small bowel were isolated and studied using physiological assessment for endothelium dependent vasorelaxation in response to Ach $\left(10^{-9}-10^{-4} \mathrm{M}\right)$ following partial constriction with endothelin-1 using previously published protocols. ${ }^{8}$ Ach induced vasodilation was reduced in vessels from two areas of intestinal web strictures (maximum diameter $7(2) \%, n=3)$ compared with the adjacent normal calibre bowel microvessels (maximum diameter 85 (5)\%; $p=<0.01$ ) (fig 1D). Maximal dilation to papaverine was similar in vessels from the area of the web (86 (4.6)\%) compared with remote regions (89 (5.2)\%). Dihydroethidine and DCF-DA staining were used to assess intravital production of ROS. ${ }^{8}{ }^{10}$ Increased ROS were seen in microvessels isolated from previously irradiated areas containing the stricture compared with vessels from the adjacent preserved bowel (fig lE). Histology of the intestinal strictures demonstrated narrowing of the arteriolar lumen due to intimal thickening and muscularis propria fibrosis, which was not seen in the adjacent preserved bowel (fig lF). Immunostaining for CD34 demonstrated preserved endothelial anatomy in the strictured microvessels (fig lG). ${ }^{11}{ }^{12}$

\section{DISCUSSION}

We report the association of a local acquired microvascular dysfunction with impaired vasodilator capacity and elevated
ROS production from microvessels localised to the area of stricture formation and not from vessels isolated from adjacent histologically normal bowel segments. This association of microvascular dysfunction in areas of stricture formation in a patient with recurrent small bowel obstruction following radiation therapy provides a novel insight into the pathophysiology of radiation enteritis. This patient presented with a two year history of gastrointestinal dysfunction following successful radiation therapy for anal squamous cell carcinoma. The patient presented with two episodes of small bowel obstruction requiring resection within 18 months of treatment. We speculate that the vascular dysfunction was localised to the endothelium as only impaired dilation to Ach (an endothelial dependent vasodilator) and not to papaverine (an endothelial independent vasodilator) was affected.

Radiation therapy is an effective modality for pelvic tumours but may be associated with gastrointestinal complications. Radiation induced complications in tissues have been carefully calculated defining dose-effect relationships (nominal standard dose/tolerance dose). The minimal and maximal tolerance doses $\left(\mathrm{TD}_{5 / 5}\right.$ and $\left.\mathrm{TD}_{50 / 5}\right)$ are 4500 $6500 \mathrm{cGy}$, respectively, for human small bowel injury, describing $5-50 \%$ rates of injury within five years following exposure. ${ }^{13}$ Chronic radiation injury to the intestine contributes to fibrosis and vascular insufficiency (chronic ischaemia). ${ }^{414}$ The rates of radiation induced bowel adverse reactions range between $5 \%$ and $25 \% .{ }^{4}$ Grossly, the intestine with chronic radiation injury has been described as fibrotic with adhesions and a mottled appearance. ${ }^{4}$ The mucosa can appear pale and telangiectatic. Consistent with this report, the area of radiation injury is multifocal with relatively normal appearing segments of bowel located immediately adjacent to the web. Taken together, this line of evidence suggests radiation induced bowel injury is a localised vascular phenomenon. ${ }^{4}{ }^{15}$ The most common portions of the intestine involved reflect the clinical application of radiation therapy and include caecum, terminal ileum, rectum, and distal sigmoid. ${ }^{16}{ }^{17}$

Vascular endothelium plays an essential role in the regulation of vascular tone and tissue perfusion through release of nitric oxide, prostacyclin, and endothelial derived hyperpolarising factor, in response to physiological stimuli, including Ach. ${ }^{8}$ Microvascular dysfunction has been identified in human disease states, including diabetes mellitus, hyperlipidaemia, and inflammatory bowel disease, and is characterised by impaired endothelium dependent vasodilation. ${ }^{8}$ Interestingly, chronic microscopic radiation induced changes are localised to the submucosa. In contrast, the pathological effects of acute radiation induced injury are observed in the mucosal layer. ${ }^{15}$ The normal submucosa is characterised by atypical fibroblast and collagen proliferation. Small arteries show hyalinised thickening of the wall with intimal foam cell proliferation and recanalised fibrin thrombi within the lumen.

The present study demonstrates that radiation induced bowel injury is also associated with an acquired local microvascular dysfunction which manifests as impaired vasodilator capacity. We hypothesise that this local microvascular dysfunction initiated by chronic elevations in ROS production following external beam ionising radiation predisposes the bowel to ischaemia and ultimately web formation. In addition, these data suggest that ROS formation is a therapeutic target in long term radiation effects on bowel, potentially preventing the impaired perfusion and subsequent web formation. Further studies investigating the short and long term effects of therapeutic radiation (and chemotherapy) on the human gut microvasculature and associated bowel complications are warranted. 


\section{ACKNOWLEDGEMENTS}

We thank Professor John E Moulder for his expertise in reviewing this manuscript and Heather Brandenburg for expert secretarial assistance.

This study was supported by a Career Development Award Grant from Crohn's Colitis Foundation of America (OAH) and Alvin and Marion Birnschein Foundation (MFO and DGB).

\section{Authors' affiliations}

O A Hatoum, S A Phillips, Division of Cardiovascular Medicine, Medical College of Wisconsin, Milwaukee, WI, USA

D G Binion, C O'Loughlin, Division of Gastroenterology and Hepatology, Medical College of Wisconsin, Milwaukee, WI, USA

R A Komorowski, Department of Pathology, Medical College of Wisconsin, Milwaukee, WI, USA

D D Gutterman, Division of Cardiovascular Medicine, Froedtert Memorial Lutheran Hospital, and Zablocki VA Medical Center, Medical College of Wisconsin, Milwaukee, WI, USA

M F Otterson, Department of Surgery, Froedtert Memorial Lutheran Hospital, and Zablocki VA Medical Center, Medical College of Wisconsin, Milwaukee, WI, USA

Conflict of interest: None declared.

Correspondence to: $\mathrm{Dr} O \mathrm{~A}$ Hatoum, Division of Cardiovascular Medicine, Medical College of Wisconsin, Milwaukee, WI 53226, USA; ohatoum@mail.mcw.edu

Revised version received 10 August 2005

Accepted for publication 11 August 2005

Published online first 26 August 2005

\section{REFERENCES}

1 Allison MC, Howatson AG, Torrance CJ, et al. Gastrointestinal damage associated with the use of nonsteroidal antiinflammatory drugs. N Engl J Med 1992;327:749-54.
2 Bjarnason I, Hayllar J, MacPherson AJ, et al. Side effects of nonsteroidal antiinflammatory drugs on the small and large intestine in humans. Gastroenterology 1993;104:1832-47.

3 Lang J, Price $A B$, Levi $A J$, et al. Diaphragm disease: pathology of disease of the small intestine induced by non-steroidal anti-inflammatory drugs. J Clin Pathol 1988;41:516-26.

4 Coia LR, Myerson RJ, Tepper JE. Late effects of radiation therapy on the gastrointestinal tract. Int J Radiat Oncol Biol Phys 1995;31:1213-36.

5 Cox JD, Byhardt RW, Wilson JF, et al. Complications of radiation therapy and factors in their prevention. World J Surg 1986;10:171-88.

6 Lucarotti ME, Mountford RA, Bartolo DC. Surgical management of intestinal radiation injury. Dis Colon Rectum 1991;34:865-9.

7 Otterson MF, Lundeen SJ, Spinelli KS, et al. Radiographic underestimation of small bowel stricturing Crohn's disease: a comparison with surgical findings. Surgery 2004; 136:854-60.

8 Hatoum OA, Binion DG, Otterson MF, et al. Acquired microvascular dysfunction in inflammatory bowel disease: Loss of nitric oxide-mediated vasodilation. Gastroenterology 2003;125:58-69.

9 Miura H, Gutterman DD. Human coronary arteriolar dilation to arachidonic acid depends on cytochrome P-450 monooxygenase and Ca2+-activated $\mathrm{K}+$ channels. Circ Res 1998:83:501-7.

10 Benov L, Sztejnberg L, Fridovich I. Critical evaluation of the use of hydroethidine as a measure of superoxide anion radical. Free Radic Biol Med 1998;25:826-31.

11 Hristov M, Erl W, Weber PC. Endothelial progenitor cells: mobilization, differentiation, and homing. Arterioscler Thromb Vasc Biol 2003;23:1185-9.

12 Hristov M, Erl W, Weber PC. Endothelial progenitor cells: isolation and characterization. Trends Cardiovasc Med 2003;13:201-6.

13 Rubin P, Casarett GW. Concepts of clinical radiation pathology. In: Dalrymple GV, Gaulden ME, Kollmorgen GM, et al. Medical radiation biology. Philadelphia: WB Saunders Company, 1973:160-89.

14 Berthrong M. Pathologic changes secondary to radiation. World J Surg 1986;10:155-70.

15 Fajardo LF, Berthrong M. Vascular lesions following radiation. Pathol Annu 1988;23:297-330.

16 Brick IB. Effects of million volt irradiation on the gastrointestinal tract. AMA Arch Intern Med 1955;96:26-31.

17 Green N, Iba G, Smith WR. Measures to minimize small intestine injury in the irradiated pelvis. Cancer 1975;35:1633-40. 